\title{
Hyperglycemia inhibits endothelial nitric oxide synthase activity by posttranslational modification at the Akt site
}

\author{
Xue Liang Du, ${ }^{1}$ Diane Edelstein, ${ }^{1}$ Stefanie Dimmeler, ${ }^{2}$ Qida Ju,,${ }^{1}$ Chengyu Sui, ${ }^{1}$ \\ and Michael Brownlee ${ }^{1}$ \\ ${ }^{1}$ Diabetes Research Center, Albert Einstein College of Medicine, Bronx, New York, USA \\ ${ }^{2}$ Molecular Cardiology, Department of Internal Medicine IV, University of Frankfurt, Frankfurt, Germany \\ Address correspondence to: Michael Brownlee, Diabetes Research Center, F-531, Albert Einstein College of Medicine, \\ Bronx, New York 10461, USA. Phone: (718) 430-3636; Fax: (718) 430-8570; E-mail: brownlee@aecom.yu.edu.
}

\begin{abstract}
Endothelial nitric oxide synthase (eNOS) is activated by phosphorylation of serine 1177 by the protein kinase Akt/PKB. Since hyperglycemia-induced mitochondrial superoxide overproduction increases $O$ linked $\mathrm{N}$-acetylglucosamine modification and decreases $O$-linked phosphorylation of the transcription factor Sp1, the effect of hyperglycemia and the hexosamine pathway on eNOS was evaluated. In bovine aortic endothelial cells, hyperglycemia inhibited eNOS activity $67 \%$, and treatment with glucosamine had a similar effect. Hyperglycemia-associated inhibition of eNOS was accompanied by a twofold increase in $O$-linked $\mathrm{N}$-acetylglucosamine modification of eNOS and a reciprocal decrease in $O$-linked serine phosphorylation at residue 1177. Both the inhibition of eNOS and the changes in its post-translational modifications were reversed by antisense inhibition of glutamine:fructose-6-phosphate amidotransferase, the rate-limiting enzyme of the hexosamine pathway, or by blocking mitochondrial superoxide overproduction with uncoupling protein-1 (UCP-1) or manganese superoxide dismutase (MnSOD). Immunoblot analysis of cells expressing myc-tagged wild-type human eNOS confirmed the reciprocal increase in $O$-linked $\mathrm{N}$-acetylglucosamine and decrease in $O$-linked serine 1177 phosphorylation in response to hyperglycemia. In contrast, when myc-tagged human eNOS carried a mutation at the Akt phosphorylation site (Ser1177), O-linked $\mathrm{N}$-acetylglucosamine modification was unchanged by hyperglycemia and phospho-eNOS was undetectable. Similar changes in eNOS activity and covalent modification were found in aortae from diabetic animals. Chronic impairment of eNOS activity by this mechanism may partly explain the accelerated atherosclerosis of diabetes.
\end{abstract}

J. Clin. Invest. 108:1341-1348 (2001). DOI:10.1172/JCI200111235.

\section{Introduction}

Diabetes-associated atherosclerosis is a major clinical problem. Diabetics have a two- to fourfold increased incidence of coronary disease and stroke, and a tenfold increased incidence of lower extremity arterial disease (1). Clinical studies have demonstrated that hyperglycemia is a major independent risk factor for diabetic macrovascular disease (2-5). Normal endothelial production of nitric oxide plays an important role in preventing vascular disease. In addition to its function as an endogenous vasodilator, nitric oxide released from endothelial cells is a potent inhibitor of platelet aggregation and adhesion to the vascular wall. Endothelial nitric oxide also controls the expression of proteins involved in atherogenesis, decreasing expression of the chemoattractant protein MCP-1, and of surface adhesion molecules such as CD11/CD18, Pselectin, VCAM-1, and ICAM-1. Endothelial cell nitric oxide also reduces vascular permeability and decreases the rate of oxidation of LDL to its proatherogenic form. Finally, endothelial cell nitric oxide inhibits proliferation of vascular smooth muscle cells (6). Endothelium-dependent vasodilatation is impaired in both microcirculation and macrocirculation during acute hyperglycemia in both normal subjects $(7,8)$ and diabetic patients $(9,10)$, suggesting that nitric oxide syn- thase (NOS) activity may be chronically impaired in diabetic patients. The mechanism by which hyperglycemia inhibits NOS activity is unknown.

Four major biochemical pathways of hyperglycemic vascular damage and hyperglycemia-induced activation of NF- $\mathrm{KB}$ have recently been shown to result from a single common mechanism: hyperglycemia-induced overproduction of superoxide by mitochondria (11, 12). Activation of the hexosamine pathway by this mechanism was found to increase $O$-linked $\mathrm{N}$-acetylglucosamine (GlcNAc) modification, and decrease $O$ linked phosphorylation of the transcription factor Sp1 (12). Because endothelial NOS (eNOS) is activated by phosphorylation of serine 1177 (Ser1177) by the protein kinase Akt/PKB $(13,14)$, the effect of hyperglycemia and the hexosamine pathway on eNOS activity and $O$-linked modification at this site was evaluated.

\section{Methods}

Materials. Eagle's MEM, nonessential amino acids, and antibiotics were from Life Technologies Inc. (Grand Island, New York, USA). FBS was from HyClone Laboratories (Logan, Utah, USA). Glucosamine, wortmannin, and monoclonal anti-phosphoserine antibodies were purchased from Sigma Chemical Co. (St. Louis, Missouri, USA). Polyclonal anti-glutamine:fructose-6- 


\section{Figure 1}

Effect of hyperglycemia and increased glucosamine on eNOS activity in BAECs. Cells were incubated with either $5 \mathrm{mM}$ glucose, 30 $\mathrm{mM}$ glucose, $5 \mathrm{mM}$ glucose plus two concentrations of glucosamine $(\mathbf{a})$, or $30 \mathrm{mM}$ glucose plus antisense, inverse, or scrambled oligonucleotides for GFAT (b). ${ }^{*} P<0.01$ compared with cells incubated in $5 \mathrm{mM}$ glucose alone. $n=5$ for each group.
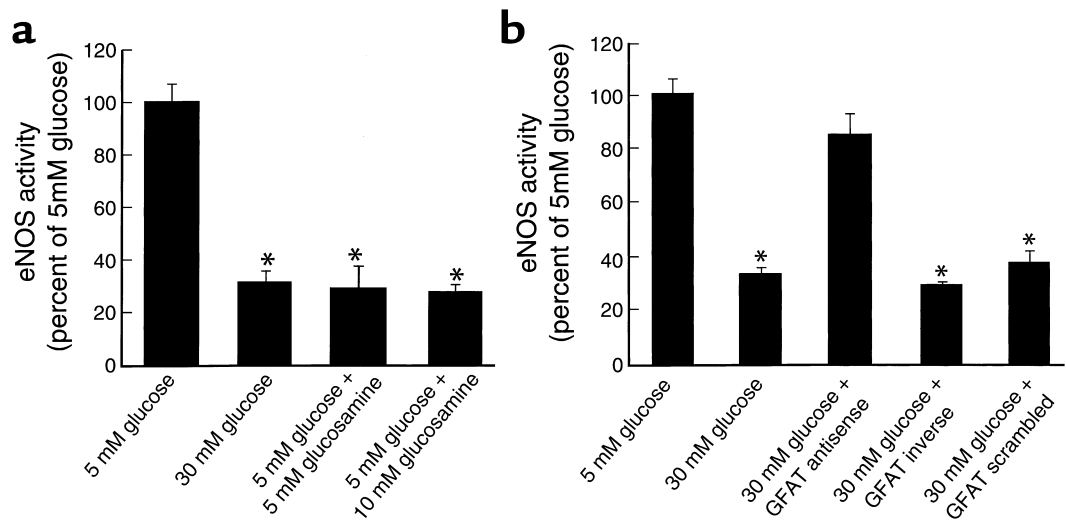

phosphate amidotransferase (GFAT) antiserum was a kind gift of Erwin Schleicher (Tubingen, Germany). Monoclonal anti-O-linked GlcNAc antibodies (RL2) were from Affinity BioReagents Inc. (Golden, Colorado, USA). Anti-phospho-eNOS(Ser1177) was from Cell Signaling Technology Inc. (Beverly, Massachusetts, USA). Anti-eNOS IgG was from Santa Cruz Biotechnology Inc. (Santa Cruz, California, USA). Polyclonal antimitochondrial antibody was from Upstate Biotechnology Inc. (Lake Placid, New York, USA). Protein A-Sepharose, $\left[{ }^{3} \mathrm{H}\right] \mathrm{L}$-arginine monohydrochloride, poly $(\mathrm{dI}-\mathrm{dC})$, and poly $\mathrm{dC}$ were obtained from Amersham Pharmacia Biotech (Piscataway, New Jersey, USA).

Cell-culture conditions. Bovine aortic endothelial cells (BAECs; passages 4-10) were cultured to confluence in Eagle's MEM containing 10\% FBS, essential and nonessential amino acids, and antibiotics. Cells were incubated with either $5 \mathrm{mM}$ glucose, $5 \mathrm{mM}$ glucose plus $5 \mathrm{mM}$ glucosamine, $10 \mathrm{mM}$ glucosamine, or 500 $\mathrm{nM}$ wortmannin, as indicated; or with $30 \mathrm{mM}$ glucose, $30 \mathrm{mM}$ glucose plus GFAT oligonucleotides (antisense, inverse, or scrambled), uncoupling protein-1 (UCP-1), or manganese superoxide dismutase (MnSOD) recombinant adenovirus, as indicated. Experiments were also performed in which cells overexpressing UCP-1 or MnSOD were cultured in $30 \mathrm{mM}$ glucose plus $10 \mathrm{mM}$ glucosamine. Cells were transfected with plasmids encoding either myc-tagged wild-type human eNOS or myc-tagged human eNOS mutated at Ser1177 (mt S1177A) and incubated in either $5 \mathrm{mM}$ glucose or 30 $\mathrm{mM}$ glucose, as indicated.

Oligonucleotide synthesis and treatment of cells. Phosphorothioate oligonucleotides were synthesized by Operon Technologies Inc. (Alameda, California, USA). The S-antisense GFAT had the following sequence: $5^{\prime}$ cCACCTGCAAGACCATcG-3' (15). Inverse and scrambled oligonucleotides were used as controls. Oligonucleotide $(36.3 \mu \mathrm{l})$ was mixed with $16.3 \mu \mathrm{l}$ polyethyleneimine per $\mathrm{ml}$ of media (16) to give a final concentration of oligonucleotide of $12.5 \mu \mathrm{M}$. Three hundred microliters of this solution was added to each well containing 200,000 cells. The cells were incubated in a $\mathrm{CO}_{2}$ incubator at $37^{\circ} \mathrm{C}$ for 2 hours. The oligonucleotide solution was then removed, the cells were washed with PBS, and fresh media was added. The cells were analyzed for eNOS activity 48 hours later.

Recombinant adenoviruses. Rat sense and antisense UCP-1 cDNA was provided by D. Riquier (Centre Nationale de la Recherche Scientifique, Unite Propre 1511, Meudon, France). Human MnSOD cDNA was provided by L. Oberley, University of Iowa College of Medicine. These cDNAs were cloned into the shuttle vector Ad5CMVK-NpA. Adenoviral vectors were prepared by the University of Iowa Gene Transfer Vector Core. Two microliters of either adenoUCP-1 or adenoMnSOD was added to 200,000 cells in a 24-well plate $(\mathrm{moi}=500)$. The cells were incubated in a $\mathrm{CO}_{2}$ incubator at $37^{\circ} \mathrm{C}$ for 90 minutes. The media containing the virus was then removed and fresh media was added.

eNOS activity. eNOS activity in cells was determined as previously described (17). In brief, cells were first incubated in L-arginine-deficient, serum-free MEM media for 6 hours. This media was then replaced with PBS buffer containing $120 \mathrm{mM} \mathrm{NaCl}, 4.2 \mathrm{mM} \mathrm{KCl}, 2.5 \mathrm{mM} \mathrm{CaCl}_{2}$, $1.3 \mathrm{mM} \mathrm{MgSO}_{4}, 1.2 \mathrm{mM} \mathrm{Na}_{2} \mathrm{HPO}_{4}, 0.37 \mathrm{mM} \mathrm{KH}_{2} \mathrm{PO}_{4}, 10$ $\mathrm{mM}$ HEPES, and $7.5 \mathrm{mM}$ glucose $(500 \mu \mathrm{l} /$ well $)$; cells were then incubated for 15 minutes at $37^{\circ} \mathrm{C}$. The eNOS activity assay was initiated by incubating cells with PBS buffer (400 $\mu \mathrm{l} /$ well) containing $\left.1.5 \mathrm{Ci} / \mathrm{ml}{ }^{3} \mathrm{H}\right] \mathrm{L}$-arginine for 15 minutes. The reaction was stopped by adding $1 \mathrm{~N}$ ice-cold TCA $(500 \mu \mathrm{l} /$ well $)$. Cytosol preparations were transferred to ice-cold silanized glass tubes and extracted three times with water-saturated ether. The samples were neutralized with $1.5 \mathrm{ml}$ of $25 \mathrm{mM}$ HEPES ( $\mathrm{pH} 8.0$ ) and applied to Dowex AG50WX8 columns (Tris form) (Sigma Chemical Co., St. Louis, Missouri, USA). Columns were eluted with $1 \mathrm{ml}$ of $40 \mathrm{mM}$ HEPES buffer ( $\mathrm{pH} 5.5$ ) containing $2 \mathrm{mM}$ EDTA and 2 mM EGTA. The eluate was collected in glass scintillation vials for $\left[{ }^{3} \mathrm{H}\right] \mathrm{L}$-citrulline quantitation by liquid scintillation spectroscopy. Activity of eNOS in cell lysates and tissue was also determined by a previously described immunoprecipitation assay (18), using the columns described above. In brief, samples were split into two tubes, one for Western blotting and one for determination of eNOS activity. eNOS immunocomplexes immobilized on protein A-Sepharose beads were resuspended in assay buffer, and eNOS activity was determined by measuring the conversion of $\left[{ }^{3} \mathrm{H}\right] \mathrm{L}$-arginine 


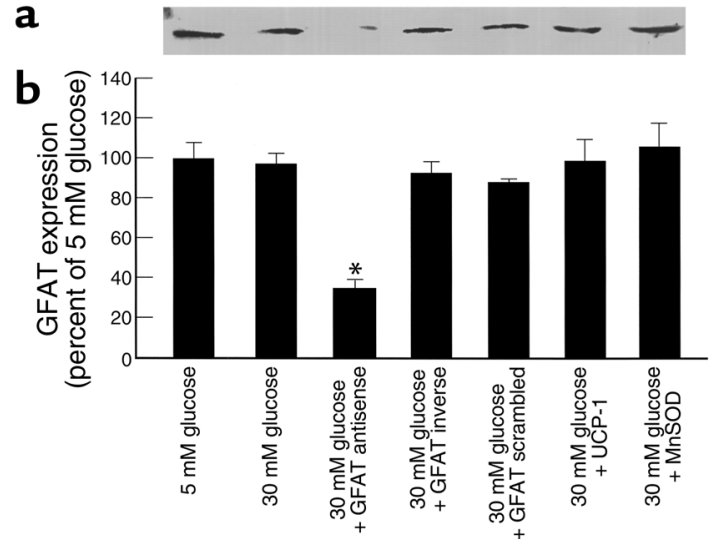

Figure 2

Effect of GFAT antisense oligonucleotides on GFAT expression. Cells were incubated with either $5 \mathrm{mM}$ glucose, $30 \mathrm{mM}$ glucose, $30 \mathrm{mM}$ glucose plus antisense, inverse, or scrambled oligonucleotides for GFAT, or $30 \mathrm{mM}$ glucose after infection with adenoviral vectors expressing either UCP-1 or MnSOD; then Western blots for GFAT were performed as described in Methods. (a) Representative Western blot. (b) GFAT expression (relative densitometric means of 4 blots). ${ }^{*} P<0.01 \mathrm{com}-$ pared with cells incubated in $5 \mathrm{mM}$ glucose.

into $\left[{ }^{3} \mathrm{H}\right] \mathrm{L}$-citrulline. Similar amounts of enzyme in each incubation were verified by eNOS blotting. All enzyme activities were corrected for $\left[{ }^{3} \mathrm{H}\right] \mathrm{L}$-arginine uptake into the cells under the various experimental conditions, determined using a previously described method (19).

$\left[{ }^{3} \mathrm{H}\right] \mathrm{L}$-arginine uptake. BAECs were incubated for 30 minutes in an uptake buffer (25 mM HEPES, $1.8 \mathrm{mM}$ $\mathrm{CaCl}_{2}, 5.4 \mathrm{mM} \mathrm{KCl}, 140 \mathrm{mM}$ choline chloride, $0.8 \mathrm{mM}$ $\mathrm{MgSO}_{4}$, and $5 \mathrm{mM}$ glucose) containing $20 \mathrm{nM}\left[{ }^{3} \mathrm{H}\right] \mathrm{L}-$ arginine. Uptake of L-arginine was terminated by adding ice-cold buffer, and cells were washed three times with $1 \mathrm{ml}$ of buffer. After the final washing, cells were lysed by the addition of $1 \mathrm{ml} 0.5 \%$ SDS in $0.1 \mathrm{~N}$ $\mathrm{NaOH}$. Cellular lysates were added to $15 \mathrm{ml}$ of Ecoscint-A scintillation fluid (Aquasol 2, Packard Instruments, Meriden, Connecticut, USA). The amount of $\left[{ }^{3} \mathrm{H}\right] \mathrm{L}$-arginine was determined by scintillation spectroscopy (LKB 1219 Rackbeta, PerkinElmer, Gaithersburg, Maryland, USA) and represented cellular transport of L-arginine.

Determination of mitochondrial membrane potential. BAECs were cultured in 24-well plates $(200,000$ cells/well) and loaded with $0.5 \mu \mathrm{M} \mathrm{JC}-1$ (Molecular Probes Inc., Eugene, Oregon, USA) in 20 mM HEPES and $0.1 \%$ BSA in MEM medium without phenol, according to the manufacturer's instructions. Samples were viewed at room temperature with a cooled CCD camera and a high-resolution Olympus IX-70 microscope (Olympus America Inc., Melville, New York, USA) with $10 \times$ objectives. Epifluorescence optics with narrow-band FITC and rhodamine filters (Chroma Technology Corp., Brattleboro, Vermont, USA) were used to detect JC-1 green fluorescence and red fluorescence from JC-1 aggregates. Digital imaging was performed with a Photometrics PXL cold CCD camera (Roper Sci- entific Inc., Tucson, Arizona, USA) run by IPLab Spectrum software (Scanalytics Inc., Fairfax, Virginia, USA) on a Power Macintosh computer. Images were acquired randomly from selected fields.

Localization of recombinant MnSOD. BAECs infected with MnSOD adenovirus were fixed with $2 \%$ paraformaldehyde for 10 minutes and postfixed for 2 minutes with methanol at $-20^{\circ} \mathrm{C}$. Cells were blocked with $20 \%$ horse serum/PBS for 30 minutes at room temperature. Cells were incubated with antimitochondrial serum and anti-MnSOD antibody for 1 hour at room temperature. Antigens were detected by immunofluorescence using anti-human IgG-Texas Red and anti-rabbit IgG-FITC conjugated secondary antibodies (Jackson ImmunoResearch Laboratories Inc., West Grove, Pennsylvania, USA). Confocal microscopic images were collected on a Radiance 2000 confocal microscope from Bio-Rad Laboratories Inc. (Hercules, California, USA) with a $\mathrm{Kr} / \mathrm{Ar}$ laser, with excitation at $488 \mathrm{~nm}$ and 568 $\mathrm{nm}$. Acousto optical tunable filter was attenuated so that there was no crosstalk from the green to red channel or vice versa. Images were taken in a single scan at 166 lines per second with a Nikon $60 \times$ NA 1.4 objective.

Immunoprecipitation. BAECs were plated in $100-\mathrm{mm}$ cell culture plates and grown to confluence. Cells $\left(2 \times 10^{7}\right)$ were scraped from the plates, pelleted, and washed twice with cold PBS. The pellet was resuspended in cold lysis buffer $(20$ mM HEPES at pH 7.9, $500 \mathrm{mM} \mathrm{NaCl}, 20 \%$ glycerol, $1 \mathrm{mM}$ DTT, $0.1 \%$ Nonidet P40, and $1 \mathrm{mM}$ PMSF) and incubated on ice for 30 minutes with gentle vortexing. Cellular debris was pelleted for 20 minutes at
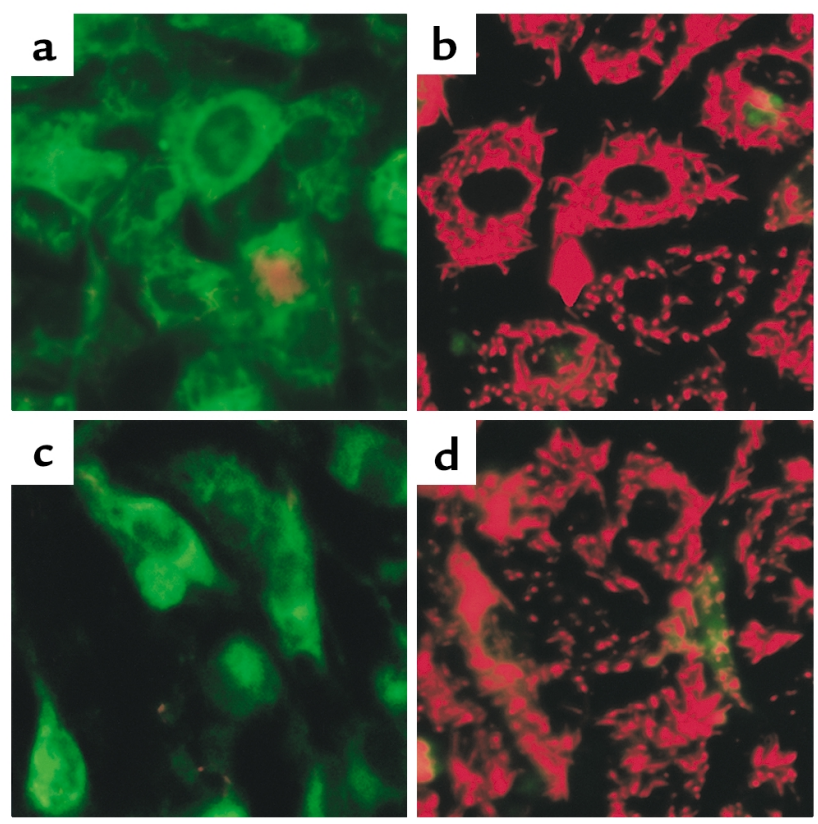

\section{Figure 3}

Digital micrographs of BAECs labeled with JC-1. (a) Cells incubated in $5 \mathrm{mM}$ glucose. (b) Cells incubated in $30 \mathrm{mM}$ glucose. (c) Cells incubated in $30 \mathrm{mM}$ glucose after infection with UCP-1-expressing adenovirus. (d) Cells incubated in $30 \mathrm{mM}$ glucose after infection with LacZ-expressing adenovirus. 


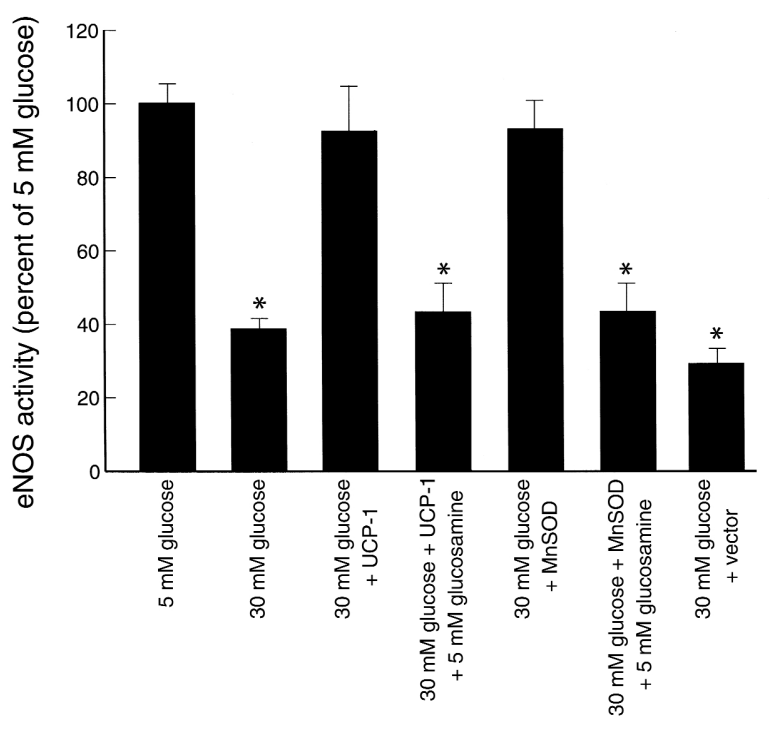

Figure 4

Effect of genes that alter mitochondrial superoxide production on eNOS activity in BAECs. Cells were incubated in $5 \mathrm{mM}$ or $30 \mathrm{mM}$ glucose alone, or in $30 \mathrm{mM}$ glucose plus either UCP-1- or MnSODexpressing adenoviral vectors, or control vector. Cells expressing UCP-1 and MnSOD were also incubated in $30 \mathrm{mM}$ glucose supplemented with $5 \mathrm{mM}$ glucosamine. eNOS activity was determined after immunoprecipitation as described in Methods. ${ }^{*} P<0.01$ compared with cells incubated in $5 \mathrm{mM}$ glucose. $n=5$ for each group.

$75,000 \mathrm{~g}$ at $4^{\circ} \mathrm{C}$. The supernatants were dialyzed at $4^{\circ} \mathrm{C}$ overnight against binding buffer $(20 \mathrm{mM}$ HEPES at $\mathrm{pH}$ 7.9, $2 \mathrm{mM} \mathrm{MgCl}_{2}, 10 \mu \mathrm{M} \mathrm{ZnSO}_{4}, 1$ mM DTT, 10\% glycerol, and $1 \mathrm{mM}$ PMSF).

Five hundred micrograms of protein was immunoprecipitated with $4 \mu \mathrm{g}$ of the indicated antibody and 20 $\mu \mathrm{l}$ of protein A-Sepharose 4B (Amersham Pharmacia Biotech) in binding buffer (final concentration $1 \mu \mathrm{g}$ protein $/ \mu \mathrm{l}$ ). The samples were rotated overnight at $4^{\circ} \mathrm{C}$. The immunoprecipitated complexes were pelleted by centrifugation $(1,000 \mathrm{~g})$ and washed $4-5$ times with binding buffer. The pellet was resuspended in $1 \times$ sample buffer, boiled, and analyzed by $7.5 \%$ SDS-PAGE with Western blotting. Aortae were homogenized in lysis buffer using a Dounce homogenizer, and were processed identically.

Western blotting. Immunoprecipitated proteins electrophoresed on 10\% PAGE gels, and cytosolic proteins electrophoresed on 12\% PAGE gels were transferred onto nitrocellulose membranes. The immunoblots were developed with 1:1,000 dilutions of the indicated antibodies, and the signal was detected with the ECL System according to the manufacturer's instructions (Amersham Pharmacia Biotech). The images were scanned into a FluorImager (Molecular Dynamics, Sunnyvale, California, USA) and analyzed using the ImageQuant 5.5 program.

Animals. Sprague-Dawley rats weighing 200 grams were fasted overnight prior to streptozotocin injection. Animals were injected with $60 \mathrm{mg} / \mathrm{kg}$ of streptozotocin dissolved in sodium citrate buffer ( $\mathrm{pH} 4.5$ ). Seventytwo hours after injection, the animals were test-bled and serum glucose was determined. Mean serum glucose was $309 \pm 8$ for the diabetic group, and $103 \pm 4$ for the nondiabetic group. Animals were killed after 16 weeks of diabetes, and the aortae were dissected and washed free of blood.

Statistics. Data were analyzed using the one-factor ANOVA procedure to compare the means of all groups. The Tukey-Kramer multiple-comparisons procedure was used to determine differences between means.

\section{Results}

Hyperglycemia inhibits eNOS activity through bexosamine pathway metabolites. eNOS activity in BAECs incubated with $30 \mathrm{mM}$ glucose for 48 hours was reduced to $32 \%$ of that in endothelial cells incubated with $5 \mathrm{mM}$ glucose (Figure 1a). Identical reductions in BAEC eNOS activity were observed when cells were incubated in 5 mM glucose supplemented with either $5 \mathrm{mM}$ or 10 mM glucosamine. Residual eNOS activity was completely blocked by the addition of $200 \mu \mathrm{M}$ L-NAME (data not shown). These observations suggested that hyperglycemia inhibited eNOS activity by increasing endogenous production of glucosamine or its metabolites via increased flux through the hexosamine pathway. To evaluate this possibility, BAECs were incubated with $30 \mathrm{mM}$ glucose alone, and with $30 \mathrm{mM}$ glucose in the presence of either antisense, inverse, or scrambled oligonucleotides for GFAT, the rate-limiting enzyme in the hexosamine pathway. In order to ensure that the results obtained using GFAT antisense oligonucleotides reflected specific downregulation of GFAT expression, Western blots were performed on cells incubated in $5 \mathrm{mM}$ glucose, $30 \mathrm{mM}$ glucose, 30 mM glucose plus GFAT antisense oligonucleotides, 30

\section{Figure 5}

Mitochondrial localization of recombinant human MnSOD. Confocal microscopic images of mitochondrial staining viewed through a Texas Red channel (a), and MnSOD signal viewed through the FITC channel (b). Colocalization of the Texas Red and FITC signals is indicated by the yellow color in the combined images (c).
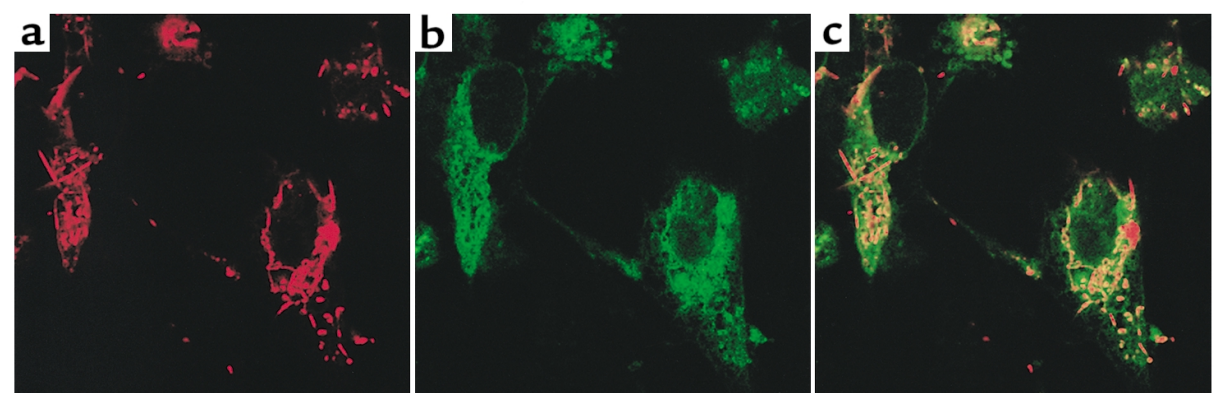
a

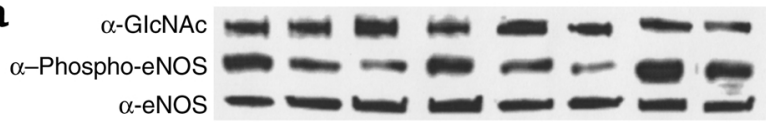

b

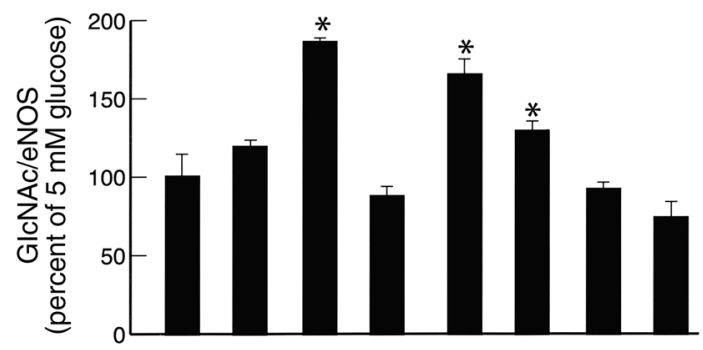

C

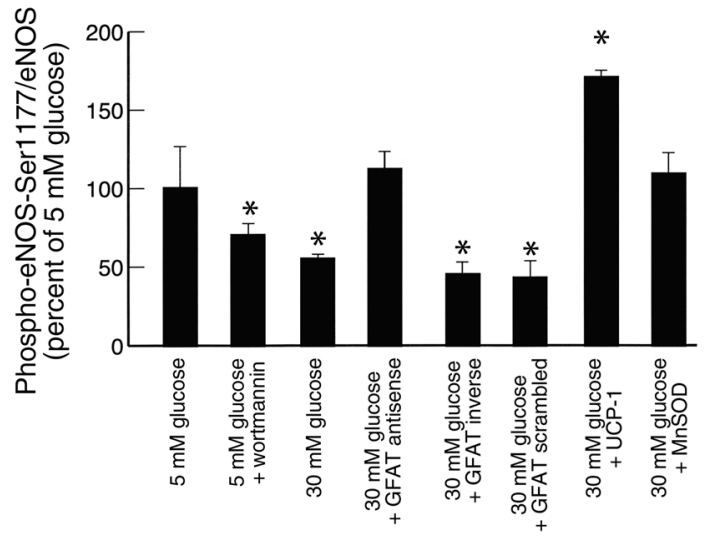

Figure 6

Effect of hyperglycemia, GFAT antisense oligonucleotides, and genes that alter mitochondrial superoxide production on eNOS O-linked GlcNAc and phospho-eNOS(Ser1177). Cells were incubated in 5 $\mathrm{mM}$ glucose alone, $5 \mathrm{mM}$ glucose plus wortmannin, $30 \mathrm{mM}$ glucose alone, and $30 \mathrm{mM}$ glucose plus either GFAT antisense, GFAT inverse, or GFAT scrambled oligonucleotides; or $30 \mathrm{mM}$ glucose plus UCP1- or MnSOD-expressing adenoviral vectors, as indicated. (a) Representative IP-Western blot. (b) eNOS O-linked GlcNAc blotted with RL2 antibody (relative densitometric means of three IP-Western blots). (c) Phospho-eNOS(Ser1177) (relative densitometric means of three IP-Western blots). ${ }^{*} P<0.01$ compared with cells incubated in $5 \mathrm{mM}$ glucose.

$\mathrm{mM}$ glucose plus GFAT inverse, and $30 \mathrm{mM}$ glucose plus GFAT scrambled oligonucleotides. GFAT expression was significantly reduced only by GFAT antisense oligonucleotides (Figure 2). GFAT antisense oligonucleotides corrected the hyperglycemia-induced inhibition of eNOS activity in BAECs to $85 \%$ of that of control (see Figure 1b), while GFAT inverse and GFAT scrambled oligonucleotides had no effect on hyperglycemia-induced eNOS inhibition. This effect of GFAT antisense oligonucleotides was abrogated by 5 $\mathrm{mM}$ glucosamine (data not shown).

Hyperglycemia inbibits eNOS activity through mitochondrial overproduction of superoxide. Because hyperglycemiainduced mitochondrial superoxide production has recently been shown to activate the hexosamine pathway (12), BAECs were incubated with $30 \mathrm{mM}$ glucose, or $30 \mathrm{mM}$ glucose plus an adenoviral vector overexpressing UCP-1. (UCP-1 is a specific protein uncoupler of oxidative phosphorylation capable of collapsing the proton electrochemical gradient that drives superoxide production [ref. 11].) In order to demonstrate directly that hyperglycemia increases the mitochondrial proton electrochemical gradient, digital imaging microscopy was performed on cells preloaded with JC-1, a dye that changes color linearly from green to red as membrane potential increases over the range of $30-180 \mathrm{mV}$. As shown in Figure 3a, BAECs incubated in $5 \mathrm{mM}$ glucose exhibit only green fluorescence. In contrast, cells incubated in $30 \mathrm{mM}$ glucose exhibit almost exclusively red fluorescence (Figure 3b). Cells incubated in $30 \mathrm{mM}$ glucose after infection with UCP-1 adenovirus exhibit only green fluorescence (Figure 3c). Cells incubated in 30 $\mathrm{mM}$ glucose after infection with adenoLacZ (Figure $3 \mathrm{~d}$ ) look identical to cells incubated in $30 \mathrm{mM}$ glucose alone. These data directly demonstrate that hyperglycemia increases the mitochondrial proton electrochemical gradient, and that UCP-1 restores it to normal. UCP-1 completely prevented the effect of hyperglycemia on the reduction in eNOS activity (Figure 4), while control vector did not.

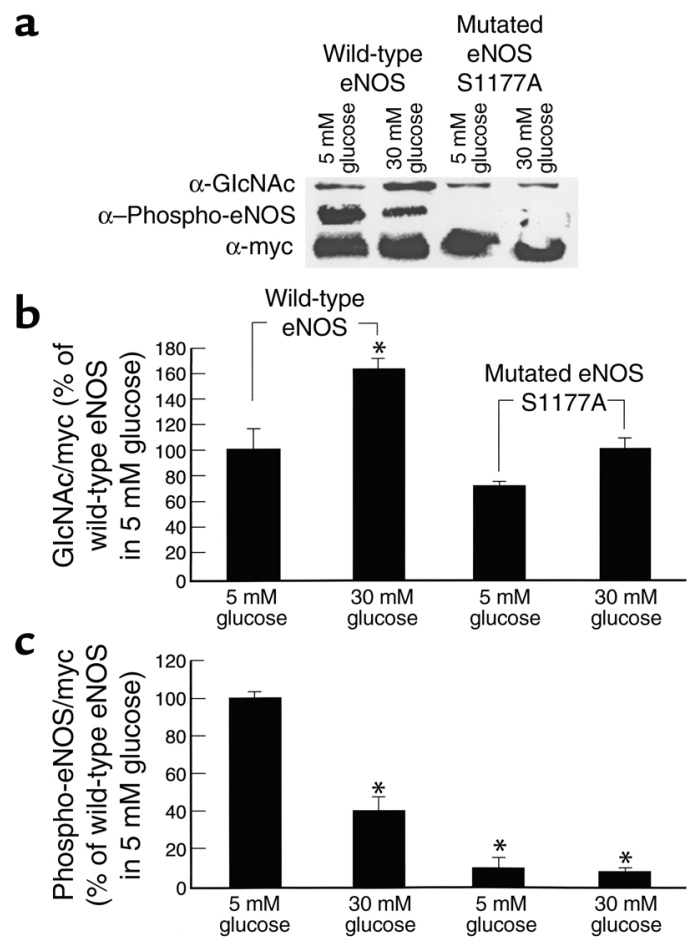

Figure 7

Effect of hyperglycemia on O-linked GlcNAc and phosphoeNOS(Ser1177) in myc-tagged wild-type human eNOS and myctagged human eNOS mutated at the Akt site. Cells were transfected as described in Methods and incubated in either $5 \mathrm{mM}$ glucose or $30 \mathrm{mM}$ glucose. Western blots were performed for O-linked GlcNAc and phospho-eNOS(Ser1177) and normalized by blotting for myc. (a) Representative IP-Western blot. (b) eNOS O-linked GlcNAc/myc expressed as percent of wild-type eNOS in $5 \mathrm{mM}$ glucose (relative densitometric means of three IP-Western blots). (c) Phospho-eNOS(Ser1177)/myc expressed as percent of wild-type eNOS in $5 \mathrm{mM}$ glucose (relative densitometric means of three IP-Western blots). ${ }^{*} P<0.01$ compared with wild-type transfected cells incubated in $5 \mathrm{mM}$ glucose. 


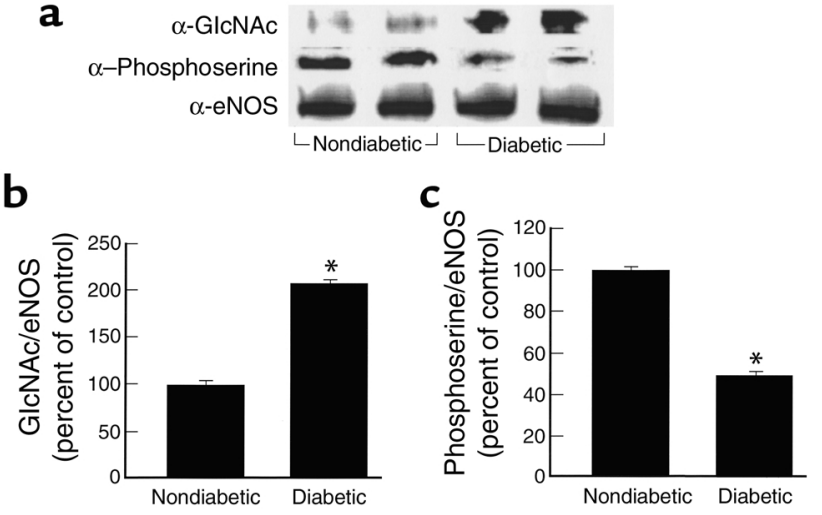

Figure 8

Effect of diabetes on aortic eNOS O-linked GIcNAc and phosphoserine. Aortae were dissected from age-matched nondiabetic and 16week streptozotocin diabetic rats. (a) Representative IP-Western blot. (b) eNOS O-linked GICNAc (relative densitometric means of three IP-Western blots). (c) eNOS O-linked phosphoserine (relative densitometric means of three IP-Western blots). ${ }^{*} P<0.01$ compared with cells incubated in $5 \mathrm{mM}$ glucose. $n=5$ for each group.

Similarly, the effect of overexpression of MnSOD, the mitochondrial form of this enzyme, which catalyzes the dismutation of superoxide to hydrogen peroxide (20), was also assessed. To confirm that the MnSOD was indeed localized to mitochondria, confocal microscopic images of mitochondrial staining viewed through a Texas Red channel, (Figure 5a) and MnSOD signal viewed through the FITC channel (Figure 5b) were obtained as described in Methods. Colocalization of the Texas Red and FITC signals is indicated by the yellow color in the combined images (Figure 5c). These results are consistent with those reported previously by $Z$ wacka et al. using the same MnSOD vector (21). Overexpression of MnSOD completely prevented the effect of hyperglycemia (Figure 4).

Correction of reduced eNOS activity by UCP-1 and MnSOD were both abrogated by the addition of $10 \mathrm{mM}$ glucosamine. Neither UCP-1 nor MnSOD changed expression levels of GFAT (Figure 2). To demonstrate directly that glycosylated eNOS has lower activity than the phosphorylated enzyme, cell lysates were immunoprecipitated for eNOS, and functional activity was then assayed (Figure 4). eNOS from cells incubated in $30 \mathrm{mM}$ glucose had $40 \%$ of the eNOS activity of cells incubated in $5 \mathrm{mM}$ glucose. eNOS activity from cells incubated in $30 \mathrm{mM}$ glucose with overexpression of either UCP-1 or MnSOD was unchanged. This protective effect on isolated eNOS activity was abrogated when cells were incubated with $5 \mathrm{mM}$ glucosamine. eNOS protein levels were unchanged by any of the conditions used. Together with the results given in Figure 1, these data show that hyperglycemia-induced mitochondrial overproduction of superoxide inhibits eNOS activity by activation of the hexosamine pathway. Hyperglycemia increases eNOS O-linked GlcNAc and decreases O-linked phosphoserine (Ser1177) in BAECs. Immunoprecipitation of eNOS followed by Western blotting (IP-Western) with antibodies to O-linked GlcNAc and phospho-eNOS(Ser1177) showed that hyperglycemia increased GlcNAc by 1.85-fold, while reciprocally decreasing phospho-eNOS(Ser1177) by $45 \%$ (Figure 6). Phosphothreonine was undetectable in these blots (data not shown). Inhibition of phosphatidylinositol 3-kinase (PI 3-kinase) by wortmannin decreased basal phospho-eNOS(Ser1177) by 30\%, consistent with the recent observations that PI 3-kinase activates eNOS by phosphorylation of Ser1177 $(13,14)$. GFAT antisense oligonucleotides, UCP-1, and MnSOD each completely prevented the hyperglycemia-induced increase in eNOS modification by GlcNAc and the reduction in phosphoeNOS(Ser1177), whereas GFAT inverse and scrambled oligonucleotides did not (Figure 6). Hyperglycemia did not change the level of eNOS protein expressed.

Hyperglycemia alters eNOS modification at the Akt phosphorylation site. In order to evaluate eNOS modification at Ser1177 by a method independent of the phosphoeNOS(Ser1177) antibody, the effects of hyperglycemia on total eNOS O-linked GlcNAc and O-linked phosphoserine at this residue were evaluated using myctagged wild-type human eNOS or myc-tagged human eNOS mutated at the Akt site (S1177A). IP-Westerns of myc-tagged wild-type human eNOS from transfected BAECs showed a reciprocal increase in $O$-linked GlcNAc and a decrease in Ser1177 O-linked phosphorylation in response to hyperglycemia (Figure 7); these changes were similar to those observed with endogenous eNOS and the phospho-eNOS(Ser1177) antibody. In contrast, modification of myc-tagged eNOS mutated at the Akt site was unchanged by hyperglycemia. Although the observed changes in O-GlcNAc and modification of the mutated construct could be indirect effects due to masking/unmasking of sites or changes in protein interactions, rather than mutation of the modification site itself, these data, together with the phospho-eNOS(Ser1177) antibody data, suggest that the observed changes do in fact reflect mutation at residue 1177 .

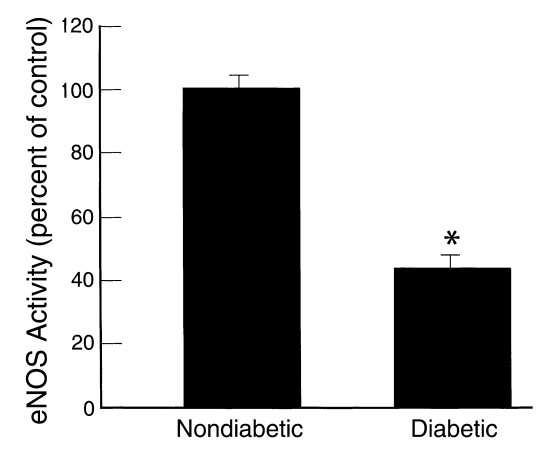

\section{Figure 9}

Effect of diabetes on aortic eNOS activity. Aortae were dissected from age-matched nondiabetic and 16-week streptozotocin diabetic rats, and eNOS activity was determined using an immunoprecipitation assay. ${ }^{*} P<0.01$ compared with cells incubated in $5 \mathrm{mM}$ glucose. $n=5$ for each group. 
Diabetes increases eNOS O-linked GlcNAc and decreases O-linked phosphoserine in rat aorta. To determine whether hyperglycemia induces the same changes in covalent modification of eNOS in vivo as it does in vitro, IP-Westerns were performed on aortic extracts from 16-week diabetic and age-matched nondiabetic rats (Figure 8). The amount of eNOS protein was unchanged by diabetes. Diabetes induced a 2.1-fold increase in GlcNAc modification of eNOS and a $50 \%$ decrease in eNOS phosphoserine; changes that were similar to the effects of hyperglycemia on eNOS in cultured BAECs (Figure 6).

Diabetes decreases eNOS activity in rat aortae. To determine whether hyperglycemia induces the same changes in eNOS activity in vivo as it does in vitro, eNOS activity was determined in aortae from 16-week diabetic and age-matched nondiabetic rats using an immunoprecipitation assay (18) (Figure 9). In diabetic aortae, eNOS activity was reduced to $43 \%$ that of controls. This decrease is of a magnitude similar to that induced by hyperglycemia in cell culture (Figure 1).

\section{Discussion}

One major hypothesis about how hyperglycemia causes diabetic vascular complications involves the hexosamine pathway $(15,22-24)$. Inhibition of GFAT, the rate-limiting enzyme in the conversion of glucose to glucosamine, blocks hyperglycemia-induced increases in the transcription of both TGF- $\alpha(22-24)$ and TGF- $\beta_{1}(15)$. The glucosamine-response element in the TGF- $\alpha$ promoter contains Sp1 sites $(22,23)$, and hyperglycemia increases expression of plasminogen activator inhibitor- 1 by increasing $O$-linked GlcNAc and decreasing $O$-linked phosphorylation of Sp1 (12). This change in Sp1 modification is a consequence of hyperglycemiainduced mitochondrial superoxide overproduction.

In this report, we show that hyperglycemia inhibits eNOS activity in cultured BAECs. It does this by activating the hexosamine pathway via mitochondrial overproduction of superoxide, which increases eNOS modification by GlcNAc and decreases eNOS serine phosphorylation in a reciprocal manner. This reciprocal modification appears to occur specifically at Ser1177, the Akt phosphorylation site responsible for the activation of eNOS $(13,14)$. Only the anti-phospho-eNOS antibody is specific for this residue, however - the RL2 anti-GlcNAc antibody is not. Residues other than Ser 1177 are also modified by GlcNAc, since eNOS mutated at residue 1177 still reacts with RL2. However, hyperglycemia does not increase modification at these sites. In $5 \mathrm{mM}$ glucose, inhibition of PI 3-kinase by wortmannin decreased phosphoeNOS(Ser1177) as expected $(13,14)$. There was little reciprocal increase in GlcNAc modification, however, suggesting that hyperglycemia-induced increases in hexosamine pathway flux are required for increased modification of eNOS by GlcNAc. In aortae from diabetic animals, changes in both covalent modification of eNOS and eNOS activity resembled those seen in BAECs cultured in $30 \mathrm{mM}$ glucose. This is the first report of eNOS modification by GlcNAc, and the first example of functional alterations in a cytoplasmic enzyme induced by this modification. Many other nuclear and cytoplasmic proteins are dynamically modified by $O$-GlcNAc moieties, and may exhibit reciprocal modification by phosphorylation (reviewed in ref. 25). Although the observations were made in macrovascular endothelial cells, and thus are directly relevant to accelerated atherosclerosis, the same mechanism most likely explains the reduced eNOS activity reported in diabetic microvessels as well $(26,27)$.

These data are consistent with previous observations implicating reactive oxygen species in impaired endothelium-dependent relaxation in response to acetylcholine in aortic rings from diabetic animals (28). Addition of either L-arginine, the substrate for eNOS, or tetrahydrobiopterin, the cofactor for eNOS, partially normalizes impaired endothelium-dependent relaxation in response to acetylcholine in diabetic arteries $(29,30)$. Since we were able to normalize eNOS activity with GFAT antisense oligonucleotides without adding either L-arginine or tetrahydrobiopterin, it may be that hyperglycemia-induced eNOS modification by GlcNAc inhibits enzyme activity by increasing the $\mathrm{K}_{\mathrm{m}}$ for one or both of these molecules. Physiologic concentrations of insulin can increase eNOS gene expression, doubling eNOS mRNA, protein, and activity. This effect is inhibited by protein kinase $\mathrm{C}$ activators (31). Since hyperglycemia activates protein kinase $C$ in BAECs by increasing mitochondrial superoxide production (11), inhibition of this vascular action of insulin in vivo by hyperglycemia could further enhance the difference in eNOS activity between diabetics and nondiabetics. The data reported here provide the basis for development of new pharmacologic agents that prevent hyperglycemia-induced activation of the hexosamine pathway. Such agents may help to prevent the development and progression of diabetes-associated atherosclerosis.

\section{Acknowledgments}

This work was supported in part by grants from NIH. The University of Iowa Gene Transfer Vector Core is supported in part by NIH and the Roy J. Carver Foundation.

1. National Diabetes Data Group. 1995. Diabetes in America. 2nd edition. National Institute of Diabetes and Digestive and Kidney Diseases. NIH publication 95-1468. Bethesda, Maryland, USA. 782 pp.

2. Singer, D.E., Nathan, D.M., Anderson, K.M., Wilson, P.W., and Evans, J.C. 1992. Association of HbA1c with prevalence of cardiovascular disease in the original cohort of the Framingham Study. Diabetes. 41:202-208.

3. Laakso, M. 1999. Hyperglycemia and cardiovascular disease in type 2 diabetes. Diabetes. 48:937-942.

4. Jensen-Urstad, K.J., Reichard, P.G., Rosfors, J.S., Lindblad, L.E., and Jensen-Urstad, M.T. 1996. Early atherosclerosis is retarded by improved long-term blood glucose control in patients with IDDM. Diabetes. 45:1253-1258.

5. Turner, R.C., et al. 1998. Risk factors for coronary artery disease in noninsulin dependent diabetes mellitus: United Kingdom Prospective Diabetes Study (UKPDS:23). BMJ. 316:823-828.

6. Li, H., and Forstermann, U. 2000. Nitric oxide in the pathogenesis of vascular disease. J. Pathol. 190:244-254.

7. Akbari, C.M., et al. 1998. Endothelium-dependent vasodilatation is impaired in both microcirculation and macrocirculation during acute hyperglycemia. J. Vasc. Surg. 28:687-694. 
8. Williams, S.B., et al. 1998. Acute hyperglycemia attenuates endotheliumdependent vasodilation in humans in vivo. Circulation. 97:1695-1701.

9. Luscher, T.F., Tanner, F.C., Tschudi, M.R., and Noll, G. 1993. Endothelial dysfunction and coronary artery disease. Annu. Rev. Med. 44:395-418.

10. Makimattila, S., et al. 1996. Chronic hyperglycemia impairs endothelial function and insulin sensitivity via different mechanisms in insulindependent diabetes mellitus. Circulation. 94:1276-1282.

11. Nishikawa, T., et al. 2000. Normalizing mitochondrial superoxide production blocks three pathways of hyperglycemic damage. Nature. 404:787-790.

12. Du, X.L., et al. 2000. Hyperglycemia-induced mitochondrial superoxide overproduction activates the hexosamine pathway and induces PAI-1 expression by increasing Sp1 glycosylation. Proc. Natl. Acad. Sci. USA. 97:12222-12226.

13. Fulton, D., et al. 1999. Regulation of endothelium-derived nitric oxide production by the protein kinase Akt. Nature. 399:597-601.

14. Dimmeler, S., et al. 1999. Activation of nitric oxide synthase in endothelial cells by Akt-dependent phosphorylation. Nature. 399:601-605.

15. Kolm-Litty, V., Sauer, U., Nerlich, A., Lehmann, R., and Schleicher, E.D. 1998. High glucose-induced transforming growth factor $\beta 1$ production is mediated by the hexosamine pathway in porcine glomerular mesangial cells. J. Clin. Invest. 101:160-169.

16. Boussif, O., et al. 1995. A versatile vector for gene and oligonucleotide transfer into cells in culture and in vivo: polyethylenimine. Proc. Natl. Acad. Sci. USA. 92:7297-7301.

17. Lantin-Hermoso, R.L., et al. 1997. Estrogen acutely stimulates nitric oxide synthase activity in fetal pulmonary artery endothelium. Am. J. Physiol. 273:L119-L126.

18. Garcia-Cardena, G., Fan, R., Stern, D.F., Liu, J., and Sessa, W.C. 1996 Endothelial nitric oxide synthase is regulated by tyrosine phosphorylation and interacts with caveolin-1. J. Biol. Chem. 271:27237-27240.

19. Ogonowski, A.A., et al. 2000. Effects of NO donors and synthase agonists on endothelial cell uptake of L-Arg and superoxide production. Am. J. Physiol. Cell Physiol. 278:C136-C143.

20. Manna, S.K., Zhang, H.J., Yan, T., Oberley, L.W., and Aggarwal, B.B. 1998.
Overexpression of manganese superoxide dismutase suppresses tumor necrosis factor-induced apoptosis and activation of nuclear transcription factor-kappaB and activated protein-1. J. Biol. Chem. 273:13245-13254.

21. Zwacka, R.M., et al. 1998. Redox gene therapy for ischemia/reperfusion injury of the liver reduces AP1 and NF-kappa B activation. Nat. Med. 4:698-704.

22. Daniels, M.C., et al. 1993. Glucose regulation of transforming growth factor-alpha expression is mediated by products of the hexosamine biosynthesis pathway. Mol. Endocrinol. 7:1041-1048.

23. McClain, D.A., Paterson, A.J., Roos, M.D., Wei, X., and Kudlow, J.E. 1992 Glucose and glucosamine regulate growth factor gene expression in vascular smooth muscle cells. Proc. Natl. Acad. Sci. USA. 89:8150-8154.

24. Sayeski, P.P., and Kudlow, J.E. 1996. Glucose metabolism to glucosamine is necessary for glucose stimulation of transforming growth factor-alpha gene transcription. J. Biol. Chem. 271:15237-15243.

25. Hart, G.W. 1997. Dynamic O-linked glycosylation of nuclear and cytoskeletal proteins. Annu. Rev. Biochem. 66:315-335.

26. Craven, P.A., Studer, R.K., and DeRubertis, F.R. 1995. Impaired nitric oxide release by glomeruli from diabetic rats. Metabolism. 44:695-698.

27. Terata, K., et al. 1999. Acetylcholine-induced arteriolar dilation is reduced in streptozotocin-induced diabetic rats with motor nerve dysfunction. Brit. J. Pharmacol. 128:837-843.

28. Pieper, G.M., Langenstroer, P., and Siebeneich, W. 1997. Diabeticinduced endothelial dysfunction in rat aorta: role of hydroxyl radicals. Cardiovasc. Res. 43:145-156.

29. Matsunaga, T.S., et al. 1996. Impairment of coronary blood flow regulation by endothelium-derived nitric oxide in dogs with alloxan-induced diabetes. J. Cardiovasc. Pharmacol. 28:60-67.

30. Pieper, G.M. 1997. Acute amelioration of diabetic endothelial dysfunction with a derivative of the nitric oxide synthase cofactor, tetrahydrobiopterin. J. Cardiovasc. Pharmacol. 29:8-15.

31. Kuboki, K., et al. 2000. Regulation of endothelial constitutive nitric oxide synthase gene expression in endothelial cells and in vivo. Circulation. 101:676-681. 